\title{
Utilización de un modelo compartimental de transporte de pesticidas en las prácticas de Química Agrícola y Ambiental
}

\author{
W.A. Contreras, I. Bautista-Carrascosa, A. Lidón, D. Ginestar \\ Universidad Politécnica de Valencia \\ wcontre@unipamplona.edu.co, ibautista@qim.upv.es, alidon@qim.upv.es, dginesta@mat.upv.es
}

\begin{abstract}
Los modelos computacionales son herramientas útiles en la mayoría de las áreas de conocimiento y pueden ser fácilmente utilizados para visualizar el comportamiento de sistemas complejos. En este trabajo se presenta un modelo sencillo de compartimentos para simular la dinámica de la concentración de un plaguicida en un cultivo de arroz. En el modelo se consideran diferentes compartimentos, tales como: aire, agua, plantas de arroz y sedimentos al que se acopla una columna de suelo mediante una ecuación de convecciónadvección. El modelo ha sido implementado en MATLAB ${ }^{\circledR}$ y tiene una interfaz gráfica de usuario (GUI) que permite cambiar fácilmente el valor de los diferentes parámetros del modelo y obtener representaciones gráficas de los resultados. Se proponen también diferentes posibilidades de aplicación del modelo para una clase práctica de Química Ambiental y Agrícola.

Computational models are useful tools in most of the knowledge areas and can be easily used to visualize the behaviour of complex systems. In this work, we present a simple compartmental model to simulate the dynamics of the concentration of a given pesticide in a rice crop. The model considers different compartments such as: air, water, rice plants and sediments, which are coupled with a soil column modelled by means of a convectionadvection equation. The model has been implemented in MATLAB ${ }^{\circledR}$ and has a Graphical User Interface (GuI) that allows to change easily the value of different parameters of the model and to obtain graphical representations of the results. Different possible applications of the model for a practical class of the subject Environmental and Agricultural Chemistry are proposed.
\end{abstract}

Keywords: Simulation, active learning, compartmental models fugacity, environmental pollution. 


\section{Introducción}

La agricultura intensiva es una de las actividades señalada como causante de un fuerte impacto ambiental, debido fundamentalmente a problemas de contaminación por el uso de pesticidas y fertilizantes. Los pesticidas son productos químicos, normalmente de naturaleza orgánica y sujetos a volatilización y degradación en los suelos, destinados a luchar contra las plagas, enfermedades y malas hierbas que disminuyen la producción de los cultivos agrícolas. Pueden ser insecticidas si se utilizan contra plagas de insectos, fungicidas si se utilizan contra hongos, bactericidas, herbicidas, etc.

El uso de estos productos es necesario para asegurar una producción de calidad que garantice la rentabilidad al agricultor, pero, en ocasiones, las prácticas agrícolas no adecuadas o el uso incorrecto de estas sustancias pueden ocasionar problemas de contaminación [1]. La contaminación ambiental por plaguicidas y fertilizantes en áreas de cultivo está, en gran medida, relacionada con las características toxicológicas y fisicoquímicas de estos compuestos, por lo que el conocimiento de su comportamiento en el suelo puede mejorar los criterios que se siguen en la actualidad para la aplicación de los mismos y predecir una posible contaminación del suelo y el agua.

La gran cantidad de sustancias utilizadas, la gran variedad de suelos y de sistemas de aplicación existentes hacen que sea complicado describir de forma general el comportamiento de los pesticidas. En el sistema suelo-agua-planta el destino del producto vendrá determinado por una serie de procesos físicos, químicos y biológicos cuya intensidad dependerá de las propiedades del suelo y las del pesticida.

Hasta la aparición de efectos ambientales adversos, el enfoque en el análisis de las propiedades de los pesticidas se concentraba en su efectividad para el control de plagas. Actualmente, es mucho más importante analizar su destino ambiental: toxicidad, transporte, degradación, etc. De este modo, para que un pesticida se pueda aplicar, la legislación exige, entre otros requisitos, la realización de una serie de ensayos de campo y de laboratorio que permitan evaluar su comportamiento en el medio natural.

En concreto, se examinan:

- La toxicidad de un pesticida y sus productos de degradación sobre plantas y animales.

- El destino químico y el transporte del pesticida en el suelo, el aire y el agua.

A partir de datos obtenidos experimentalmente se pueden elaborar modelos de simulación que, una vez calibrados, permiten ampliar el conocimiento sobre el sistema estudiado en situaciones que no se han medido experimentalmente. El modelado matemático como herramienta de descripción es aplicable en la mayoría de las áreas de conocimiento y permite el estudio del comportamiento del sistemas complejos. La simulación es una técnica que facilita el aprendizaje activo [2]; los alumnos adquieren el aprendizaje a través de las decisiones que toman a lo largo de la tarea y su utilización implica tres fases:

- Fase de información. Se marcan los objetivos a conseguir y los alumnos buscan la información necesaria.

- Simulación. En este caso, utilizando el programa desarrollado.

- Evaluación y análisis de la situación. Permite a los estudiantes interpretar los resultados. 
Con esta técnica el alumno aprende a interpretar las características del pesticida y del suelo, conocimiento que le va a permitir tomar decisiones acertadas en un hipotético uso futuro del mismo.

Dada la importancia del estudio de los pesticidas, se ha considerado conveniente desarrollar un modelo sencillo del transporte de pesticidas en el suelo, que pueda ser utilizado en clases prácticas de simulación. El uso de un modelo de este tipo, permite analizar el efecto de la aplicación de insecticidas y herbicidas. Para utilizar el modelo, hay que conocer las propiedades fisicoquímicas del producto estudiado. Estas características están recogidas en las etiquetas de los productos comerciales. Los alumnos de la titulación de Ingeniero Agrónomo aprenden a interpretar estas etiquetas en las clases teóricas de la asignatura Química Agrícola y Ambiental. Ésta es una asignatura obligatoria de universidad que se imparte en el tercer curso de la titulación de Ingeniero Agrónomo. Es, por tanto, una asignatura común a todas las intensificaciones de la titulación. Entre sus objetivos se encuentran el estudio y manejo de los pesticidas, conocer sus propiedades y características y la interpretación de su comportamiento en el medio ambiente. De los cinco bloques de los que consta la asignatura, el cuarto está dedicado a la Química de los plaguicidas y el quinto a los aspectos medioambientales del uso de estos productos.

El uso del modelo también requiere conocer las propiedades del suelo que el alumno ha adquirido en la asignatura Suelo, Agua y Atmósfera.

\section{Modelo de transporte de pesticidas}

Hoy día existe una gran variedad de modelos utilizados para describir el comportamiento de los plaguicidas en el sistema agua-suelo-planta. El modelo que hemos utilizado, hace uso del concepto de fugacidad, como fuerza conductora de los procesos de partición del compuesto entre los compartimentos del sistema aire, agua sólidos y planta [3].

Para desarrollar el modelo, se ha utilizado un ejemplo sencillo de cultivo con varias fases como es el cultivo de arroz inundado (figura 1), al que se le acopla una columna de suelo a través de la cual se transporta el pesticida.

En este cultivo, se pueden distinguir las fases:

- Aire (fase gaseosa).

- Agua (fase líquida).

- Planta.

- Sedimento.

- Columna de suelo.

Se consideran los compartimentos y las relaciones entre ellos que se muestran en la figura 2.

La dinámica de los distintos compartimentos se obtiene planteando ecuaciones de balance. Por ejemplo, para el caso que se muestra en la figura 3, con dos compartimentos, se tienen las siguientes ecuaciones de balance. Para el compartimento 1,

$$
\frac{d x_{1}}{d t}=\left\{\begin{array}{l}
- \text { Transferencia hacia } 2-\text { salidas hacia el exterior } \\
+ \text { entrada desde } 2+\text { entrada desde el exterior }
\end{array}\right.
$$




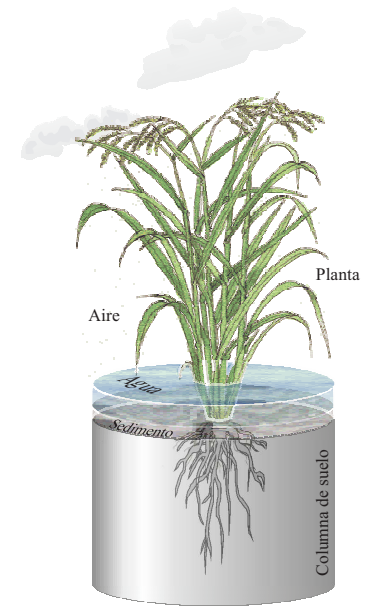

Figura 1: Distintas fases del cultivo del arroz.

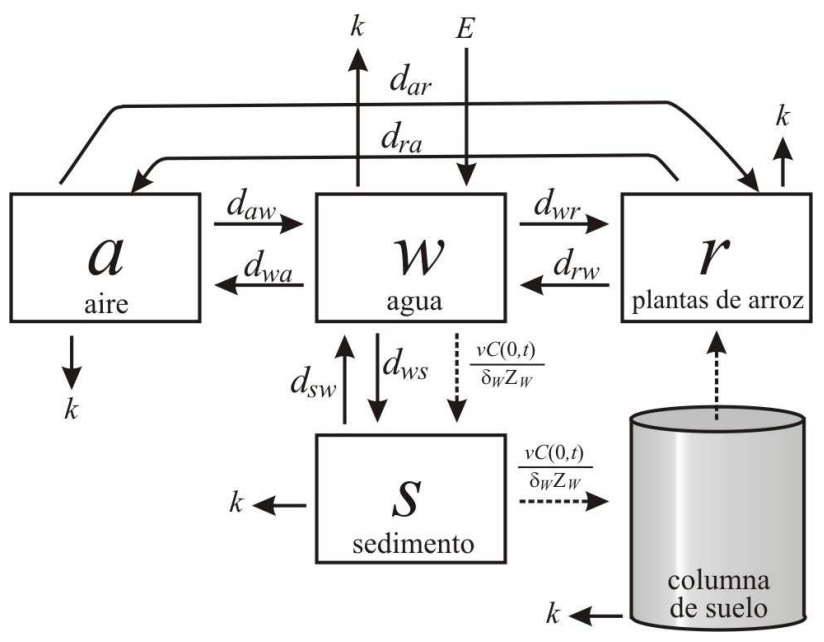

Figura 2: Compartimentos del modelo para el cultivo del arroz.

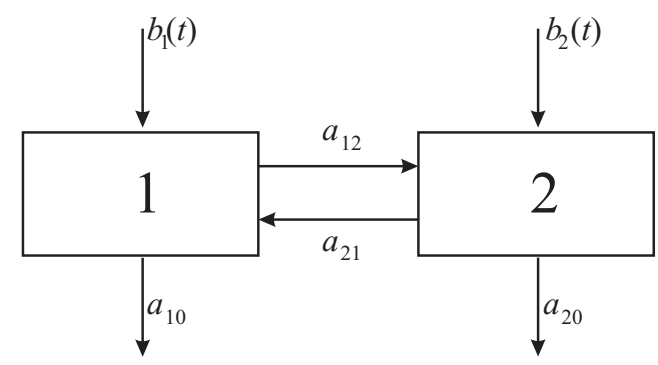

Figura 3: Estructura de dos compartimentos. 
y para el compartimento 2

$$
\frac{d x_{2}}{d t}=\left\{\begin{array}{l}
- \text { Transferencia hacia } 1-\text { salidas hacia el exterior } \\
+ \text { entrada desde } 1+\text { entrada desde el exterior }
\end{array}\right.
$$

En concreto, se hará uso de un modelo de fugacidad de nivel IV, que viene dado por un sistema de ecuaciones diferenciales que modelizan las variaciones de la fugacidad con el tiempo en cada compartimento [4]. Las concentraciones relativas de un producto químico en las fases gaseosa, líquida y sólida, $C_{i}$, pueden relacionarse con el concepto de fugacidad (presión de vapor del producto en equilibrio con una fase), $f_{i}$, mediante la expresión

$$
C_{i}=Z_{i} f_{i}
$$

donde $Z_{i}$ es la capacidad de fugacidad.

La capacidad de fugacidad del aire está definida por

$$
Z_{a}=\frac{1}{R T}
$$

donde $Z_{a}$ se mide en mol m${ }^{-3} \mathrm{~Pa}^{-1}, T$ es la temperatura del aire en $\mathrm{K}$ y $R$ es la constante de un gas perfecto e igual a $8.314 \mathrm{~m}^{3} \mathrm{~Pa} \mathrm{~mol}^{-1} \mathrm{~K}^{-1}$.

La capacidad de fugacidad en el agua está definida por

$$
Z_{w}=\frac{1}{H}
$$

donde $Z_{w}$ se mide en mol m${ }^{-3} \mathrm{~Pa}^{-1}$, y $H$ en $\mathrm{m}^{3} \mathrm{~Pa} \mathrm{~mol}^{-1}$ es la constante de Henry para el pesticida. Esta constante se puede estimar mediante la expresión

$$
H=\frac{p_{m} p_{v}}{s_{w}}
$$

donde $p_{m}\left(\mathrm{~g} \mathrm{~mol}^{-1}\right)$ es la masa molar del pesticida, $p_{v}$, en Pa, es la presión de vapor del pesticida y $s_{w}$, en $\mathrm{g} \mathrm{m}^{-3}$, es la solubilidad en el agua del pesticida.

La capacidad de fugacidad de las plantas de arroz puede ser definida por

$$
Z_{r}=\left(x_{w} Z_{w}+x_{l} k_{o w} Z_{w}\right)\left(\rho_{r} / \rho_{w}\right)
$$

donde $Z_{r}$ se mide en mol m${ }^{-3} \mathrm{~Pa}^{-1}, \rho_{w}$, en $\mathrm{kg} \mathrm{m}^{-3}$, es la densidad del agua, $k_{\text {ow }}$ es el coeficiente de partición octanol-agua del pesticida, $\rho_{r}$, en $\mathrm{kg} \mathrm{m}^{-3}$, es la densidad de las plantas de arroz, $x_{w}$ es la fracción volumétrica de agua en las plantas de arroz y $x_{l}$ es la fracción volumétrica de lípidos en la planta.

La capacidad de fugacidad del sedimento se define por

$$
Z_{s}=\frac{\rho_{s} O c_{s} k_{o c}}{H},
$$

donde $Z_{s}$ se mide en mol m${ }^{-3} \mathrm{~Pa}^{-1}, \rho_{s}$ en $\mathrm{kg} \mathrm{m}^{-3}$ es la densidad del sedimento, oc $s$ es la fracción volumétrica de carbón orgánico en el sedimento y $k_{o c}$ en $\mathrm{m}^{3} \mathrm{~kg}^{-1}$ es el coeficiente de partición del pesticida en el carbono orgánico del sedimento.

Para cada compartimento la ecuación de balance es de la forma

$$
\begin{aligned}
V_{i} Z_{i} \frac{d f_{i}}{d t}= & E_{i}+G_{i} C_{B i}-G_{i} Z_{i} f_{i}-k_{r}^{i} V_{i} Z_{i} f_{i} \\
& -\sum_{j=1, j \neq 1}^{n} d_{i j}\left(f_{i}-f_{j}\right), \quad i=1, \ldots, n,
\end{aligned}
$$


con lo que considerando los compartimentos mostrados en figura 2, se tiene el modelo,

$$
\begin{aligned}
\frac{d f_{a}}{d t}= & \frac{d_{w a} f_{w}}{V_{a} Z_{a}}+\frac{d_{r a} f_{a}}{V_{a} Z_{a}}+\frac{G_{a} C_{B a}}{V_{a} Z_{a}}-\frac{d_{a w} f_{a}}{V_{a} Z_{a}}-\frac{d_{a r} f_{a}}{V_{a} Z_{a}}-\frac{G_{a} f_{a}}{V_{a}}-\lambda_{a} f_{a}, \\
\frac{d f_{w}}{d t}= & \frac{d_{a w} f_{a}}{V_{w} Z_{w}}+\frac{d_{r w} f_{r}}{V_{w} Z_{w}}+\frac{d_{s w} f_{s}}{V_{w} Z_{w}}+\frac{G_{w} C_{B w}}{V_{w} Z_{w}}-\frac{v C(0, t)}{\delta_{w} Z_{w}} \\
& -\frac{G_{w} f_{w}}{V_{w}}-\frac{d_{w a} f_{w}}{V_{w} Z_{w}}-\frac{d_{w s} f_{w}}{V_{w} Z_{w}}-\frac{d_{w r} f_{w}}{V_{w} Z_{w}}-\lambda_{w} f_{w}, \\
\frac{d f_{r}}{d t}= & \frac{d_{w r} f_{w}}{V_{r} Z_{r}}+\frac{d_{a r} f_{a}}{V_{r} Z_{r}}+\frac{Q_{w} T_{s f c}}{\delta_{r} V_{r} Z_{r}} \int_{0}^{\delta_{r}} C(x, t) d x-\frac{d_{r w} f_{r}}{V_{r} Z_{r}}-\frac{d_{r a} f_{r}}{V_{r} Z_{r}}-\lambda_{r} f_{r}, \\
\frac{d f_{s}}{d t}= & \frac{d_{w s} f_{w}}{V_{s} Z_{s}}+\frac{G_{s} C_{B s}}{V_{s} Z_{s}}-\frac{G_{s} f_{s}}{V_{s}}-\frac{d_{s w} f_{s}}{V_{s} Z_{s}}-\lambda_{s} f_{s} .
\end{aligned}
$$

El origen y el significado de los distintos coeficientes que aparecen en las ecuaciones anteriores se puede consultar en la referencia [4] y las correspondientes que allí se citan.

Para simular el transporte del pesticida en una columna de suelo, se hace uso de una ecuación unidimensional de convección-dispersión de la forma,

$$
D_{e} \frac{\partial^{2} C}{\partial x^{2}}-v \frac{\partial C}{\partial x}-R_{f}\left(\frac{\partial C}{\partial t}-C\left(\mu-\frac{Q_{w} T_{s f c}}{V_{r}}\right)\right)=0,
$$

donde $C=C(x, t)$, en mol $\mathrm{m}^{-3}, D_{e}$ en $\mathrm{m}^{2} \mathrm{~h}^{-1}, v$ en $\mathrm{m} \mathrm{h}^{-1}, R_{f} \mathrm{y} \mu \mathrm{en} \mathrm{h}^{-1}$ son, respectivamente, la concentración del pesticida en la solución del suelo, el coeficiente efectivo de dispersión, la velocidad del agua en el poro, el factor de retardo del pesticida y la vida media del pesticida en el suelo. $T_{s f c}$ es el coeficiente de absorción radicular del pesticida por la planta, $Q_{w}$ es el caudal de agua transpirado por la planta y $V_{r}$ es el volumen radicular.

El factor de retardo del pesticida se calcula mediante la expresión

$$
R_{f}=1+\frac{\rho v_{o c} k_{o c}}{\theta}
$$

donde $\rho$, en $\mathrm{kg} \mathrm{m}^{-3}$, es la densidad del terreno, $v_{o c}$ es la fracción volumétrica de carbón orgánico en el suelo, $\theta$ es la fracción volumétrica de agua en el suelo y $k_{o c}$, en $\mathrm{m}^{3} \mathrm{~kg}^{-1}$, es el coeficiente de partición del pesticida en el carbón orgánico del suelo.

El acople de la columna de suelo con los otros compartimentos del modelo se hace por medio de las condiciones de contorno, que son de la forma

$$
\begin{aligned}
& v C(0, t)-D_{e} \frac{\partial C(0, t)}{\partial t}=v Z_{w} f_{w}(t), \quad t \geq 0 \\
& \frac{\partial C\left(z_{\infty}, t\right)}{\partial z}=0, \quad t \geq 0
\end{aligned}
$$

Para la solución numérica del modelo se utiliza el método de Crank-Nicolson [4].

\section{Aplicación didáctica}

Para la utilización del modelo en las prácticas de la asignatura de Química Agrícola, el modelo numérico desarrollado, se ha implementado en un programa de MATLAB ${ }^{\complement}$ que utiliza una serie 


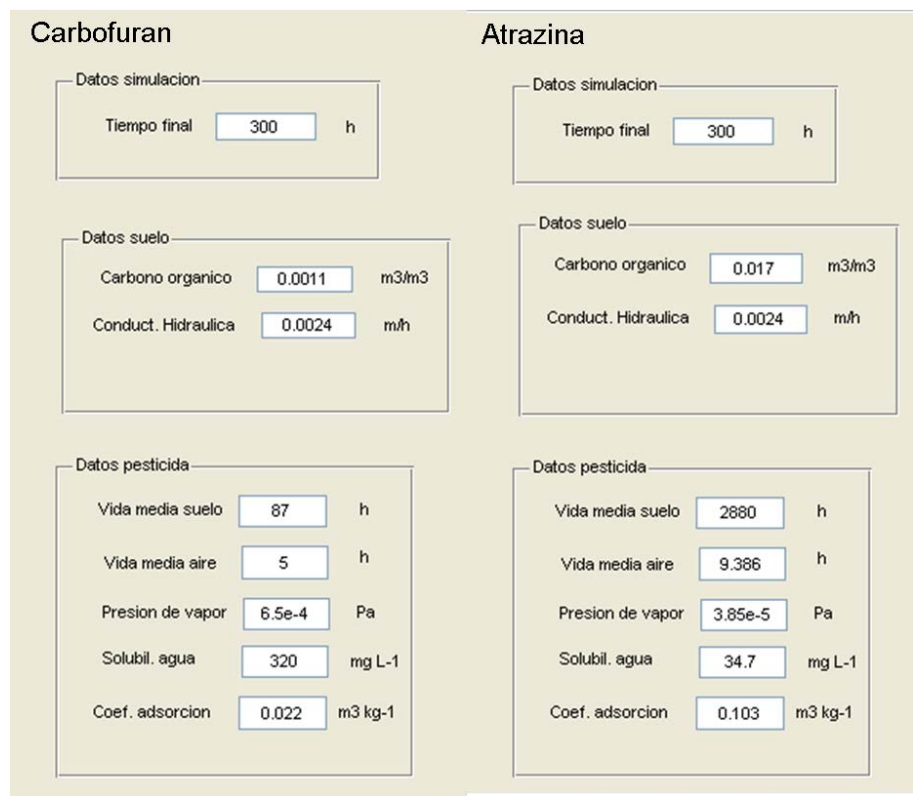

Figura 4: Datos de entrada considerados para los pesticidas carbofuran y atracina.

de ventanas (GUi) que permiten variar de forma sencilla una serie de parámetros y obtener representaciones gráficas de la solución del modelo.

El objetivo, por tanto, de la realización de la práctica es que el alumno comprenda el significado de las variables que caracterizan el comportamiento ambiental del pesticida. Son particularmente importantes propiedades del suelo como la conductividad hidráulica, que deter mina la velocidad de transporte del pesticida y el contenido de carbono orgánico que determina la mayor o menor inmovilización del pesticida en el suelo. También se pueden considerar variables del pesticida como su vida media, que determina la velocidad de degradación del pesticida, su presión de vapor, que determina la volatilización, así como la solubilidad y el coeficiente de adsorción.

Se ha particularizado el modelo para evaluar el comportamiento de dos pesticidas (atrazina y carbofuran) en suelos con diferentes características que se muestran en la figura 4.

Para ver el efecto de una propiedad importante del suelo, como es la conductividad hidráulica saturada, directamente implicada en el movimiento de solutos, se simula el comportamiento de la atrazina en un suelo con baja capacidad de conducir agua (arcilloso) y en un suelo con una conductividad hidráulica más alta (franco limoso). Para ello, se le proporcionan al alumno valores habituales de conductividad hidráulica saturada $\left(k_{s}\right)$, como los mostrados en la tabla 11.1.

Tras la simulación, se obtienen los resultados para el suelo arcilloso que se muestran en la figura 5. Para el suelo franco limoso, se obtienen los resultados mostrados en la figura 6. En los resultados obtenidos se aprecia la importancia de la conductividad hidráulica saturada del suelo sobre el transporte del pesticida. En un suelo con conductividad baja el pesticida queda retenido en los $20 \mathrm{~cm}$ superficiales del suelo al final de la simulación, en cambio en un suelo con una conductividad hidráulica saturada más alta la distancia a la que se transporta el pesticida es cinco veces superior.

De forma similar a la expuesta, el alumno puede comparar el comportamiento de dos pesticidas 
Tabla 11.1: Valores de conductividad hidráulica saturada

\begin{tabular}{lc}
\hline Clase textural & $k_{s}\left(\mathrm{~m} \mathrm{~h}^{-1}\right)$ \\
\hline Franco limosa & $6.8 \times 10^{-3}$ \\
Franco arcillosa & $2.3 \times 10^{-3}$ \\
Arcillo arenosa & $1.2 \times 10^{-3}$ \\
Franco arcillo limosa & $1.5 \times 10^{-3}$ \\
Arcillo limosa & $9.0 \times 10^{-4}$ \\
Arcillosa & $6.0 \times 10^{-4}$ \\
\hline
\end{tabular}

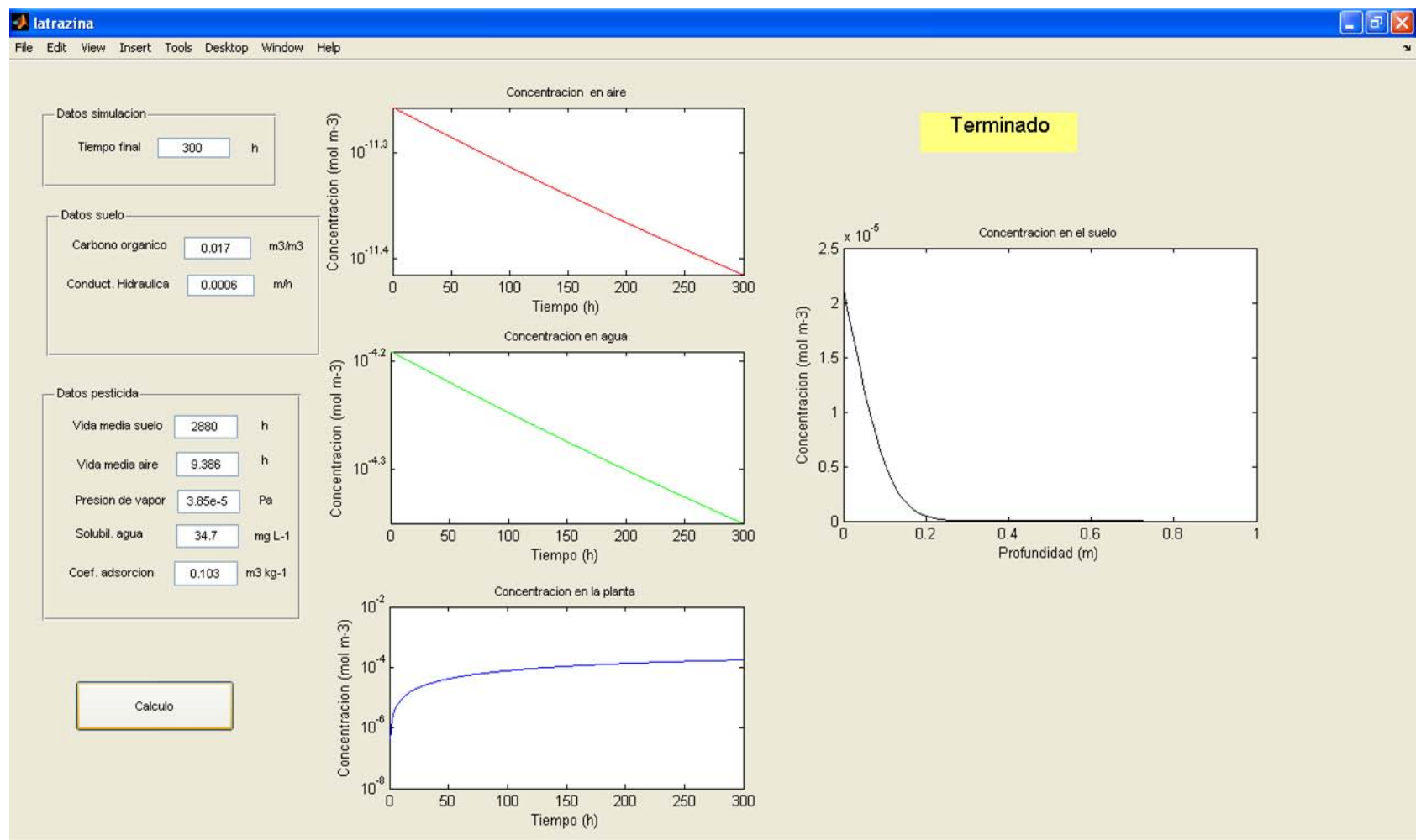

Figura 5: Resultados para el suelo arcilloso. 


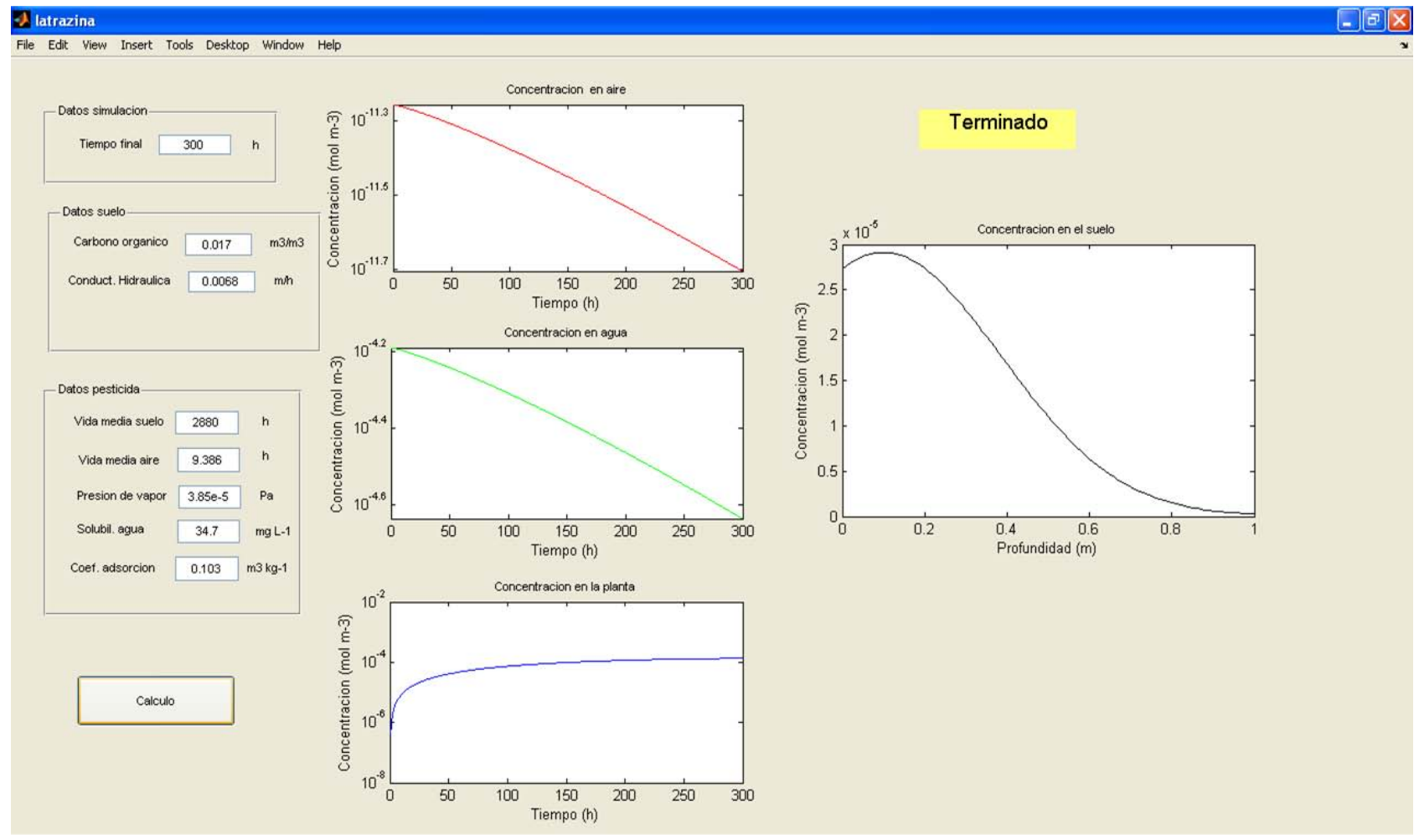

Figura 6: Resultados para el suelo franco limoso.

diferentes en un mismo suelo, o bien modificar algunas propiedades de un pesticida para evaluar su influencia en el comportamiento ambiental de este pesticida.

\section{Conclusiones}

Se ha construido un modelo sencillo para el transporte de pesticidas aplicado a una plantación de arroz. Este modelo está constituido por cuatro ecuaciones diferenciales acopladas a una ecuación de convección dispersión. Estas ecuaciones se pueden resolver mediante un esquema numérico sencillo, por lo que resulta un modelo con interés didáctico, tanto para asignaturas de tipo matemático, como agronómico.

La aplicación desarrollada va a servir a los alumnos para:

- Sintetizar la información necesaria para estudiar el comportamiento de un pesticida.

- Analizar los resultados obtenidos al ir cambiando las condiciones de la simulación.

La implementación del modelo en MATLAB $^{\circledR}$, permite la creación de una aplicación de ventanas (GUi) que facilita el uso del modelo y la interpretación de los resultados por parte de los alumnos. Este tipo de modelos pueden ser de utilidad para el diseño de actividades que serán necesarias en las nuevas metodologías propiciadas por el EEES. 



\section{Referencias}

[1] M. Laegreid, O.C. Bockman, O. Kaarstad, Agriculture, Fertilizers and Environment. CABI Publ., Wallingford and Norsk Hydro ASA. Oslo, (1999).

[2] M. A. Andreu, Simulación. En: Metodologías activas. Universidad Politécnica de Valencia, 93-105, (2008).

[3] D. Mackay, S. Paterson, Calculating fugacity. Environ. Sci. Technol., 15(9), 1006-1014, (1981).

[4] W.A. Contreras, D. Ginestar, L.C. Paraíba, R. Bru, Modelling the pesticide concentration in a rice field by a level IV fugacity model coupled with a dispersion-advection equation. Computers and Mathematics with Applications, 56, 657-669, (2008). 
